\title{
Nanometric morpho-structural characterization of mesoporous metal oxide semiconductors for chemo-resistive gas sensors
}

\author{
C. Mihalcea ${ }^{1,3}$, A. C. Kuncser ${ }^{1}$, I. F. Mercioniu ${ }^{1}$, A. M. Vlaicu ${ }^{1}$, S. Somacescu ${ }^{2}$, C. Ghica ${ }^{1}$ \\ 1 National Institute of Materials Physics, 77125 Magurele, Romania \\ 2 "Ilie Murgulescu" Institute of Physical Chemistry, 060021, Bucharest, Romania \\ 3 Faculty of Physics, University of Bucharest, 77125 Magurele, Romania
}

* Correspondence: catalina.mihalcea@infim.ro

$\mathrm{SnO}_{2}$ and $\mathrm{ZnO}$ are among the most studied semiconductor oxides for applications as gas sensing devices for detecting and monitoring the presence of toxic gases such as $\mathrm{CO}, \mathrm{NO}, \mathrm{NO}_{2}$. Enhanced sensing properties have been recently reported on gas sensor based on $\mathrm{SnO}_{2}-\mathrm{ZnO}$ composites due to the formation of hetero-junction between $\mathrm{ZnO}$ and $\mathrm{SnO}_{2}$ grains and the contribution of a depleted layer at the intergrain $\mathrm{ZnO}-\mathrm{SnO}_{2}$ interfaces. The sensing characteristics can be further improved by adding metallic nanoparticles with a catalytic activity, like Pt or Pd. Along with the electrical properties of such a complex system, morphology plays an essential role in facilitating and enhancing the interaction with the surrounding gas. In our work, $\mathrm{ZnO}$ doped $\mathrm{SnO}_{2}$ mesoporous system for gas sensors has been prepared using solvothermal methods in various experimental conditions. Aiming for a proper optimization of the gas sensing properties, the above mentioned system has been decorated with Pd using wet spray method. This work presents an in depth structural and morphological study by combined techniques of analytical electron microscopy on the mesoporous $\mathrm{SnO}_{2}-\mathrm{ZnO}$ systems decorated with $\mathrm{Pd}$ in function of the synthesis conditions. Electron tomography has been employed for a complete, 3D investigation of the $\mathrm{Pd}$ clusters distribution within the mesoporous $\mathrm{SnO}_{2}-\mathrm{ZnO}$ matrix. By a proper image segmentation $\mathrm{Pd}$ clusters have been successfully isolated in the surrounding $\mathrm{ZnO}$-doped $\mathrm{SnO}_{2}$ matrix, thus allowing more complex correlations between the micro/nano-structure and the gas sensing properties.

Keywords: mesoporous metal oxide semiconductors, gas sensors, $\mathrm{SnO}_{2}, \mathrm{ZnO}$.

\section{Funding}

Not applicable.

\section{Acknowledgments}

This work was supported by the Romanian Ministry of National Education, CNCS - UEFISCDI, through PN-III-P1-1.2-PCCDI Ctr. No. 47/2018 and Core Project PN19-03 (Ctr. No. 21 N/2019).

Cite This Work: Mihalcea, C.; Kuncser, A.C.; Mercioniu, I.F.; Vlaicu, A.M.; Somacescu, S.; Ghica, C Nanometric morpho-structural characterization of mesoporous metal oxide semiconductors for chemoresistive gas sensors. Proceedings Int 2019, 1, 0034-0035.

https://doi.org/10.33263/Proceedings 11.00340035 
Nanometric morpho-structural characterization of mesoporous metal oxide semiconductors for chemo-resistive gas sensors

\section{Conflicts of Interest}

The authors declare no conflict of interest.
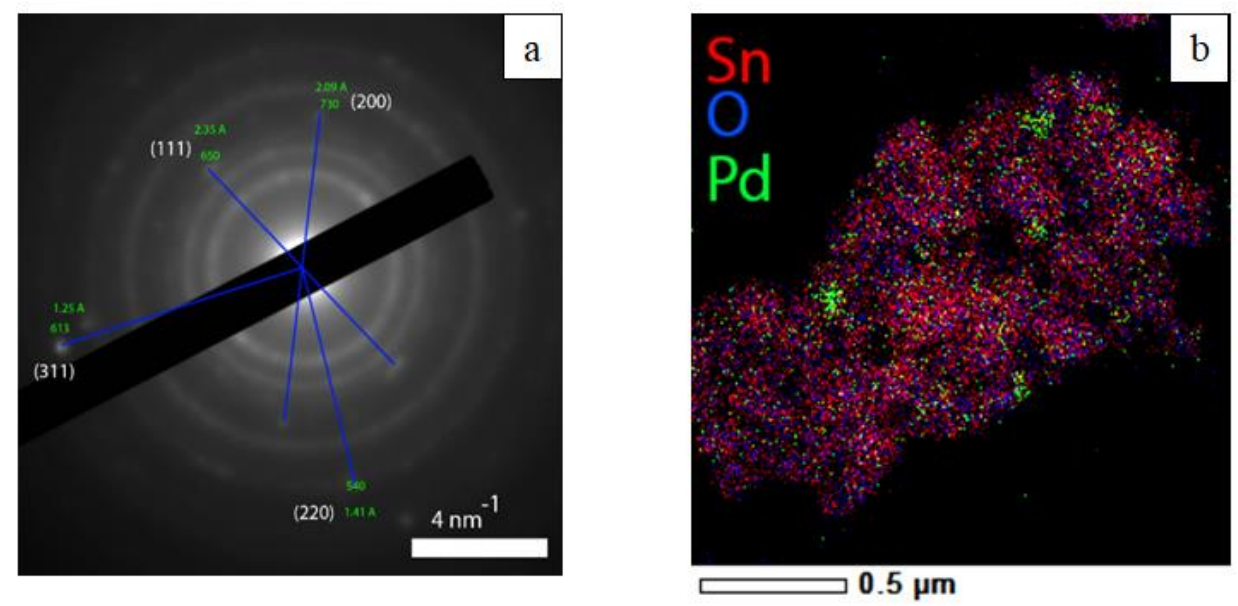

Figure 1. (a). SAED pattern taken on a region from the Pd-SnO10ZnO-Bj sample and (b) the elemental STEMEDS map showing regions having dimensions of $100 \mathrm{~nm}$, having a high amount of Pd.
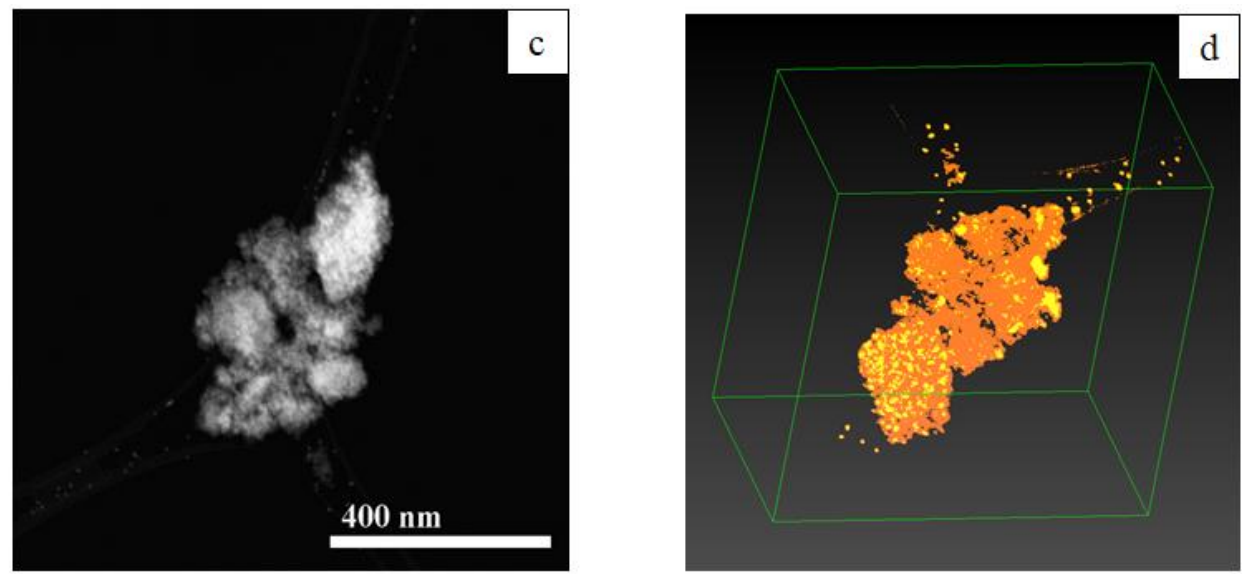

Figure 2. (c). Dark-Field STEM image taken on the Pd-Sn10Zn sample and (d) the 3D segmented reconstruction showing Pd clusters

\section{References}

1. Albanese, E.; Di Valentin, C.; Pacchioni, G.; Sauvage, F.; Livraghi, S.; Giamello, E. Nature of J. Phys. Chem. Sol. 1970, 31, 1027-1040, https://doi.org/10.1016/0022-3697(70)90313-6.

Paramagnetic Species in Nitrogen-Doped SnO2: A 3. Popa, A.; Raita, O.; Stan, M.; Pana, O.; Borodi, G.; Combined Electron Paramagnetic Resonance and Density Functional Theory Study. Giurgiu, L.M. Electron Paramagnetic Resonance of MnPhys. Chem C 2015, 119, 26895-26903, https://doi.org/10.1021/acs.jpcc.5b09613 Doped Sn1-x Mn x O2 Powders. Appl. Magn. Reson. 2012, 42, 453-462, https://doi.org/10.1007/s00723-0120319-8.

2. Simanek, E.; Muller, K.A. Covalency and hyperfine structure constant A of iron group impurities in crystals.

(C) 2019 by the authors. This article is an open access article distributed under the terms and conditions of the Creative Commons Attribution (CC BY) license (http://creativecommons.org/licenses/by/4.0/). 